\title{
Passive elimination of correlated amplitude fluctuations in ultrabroadband supercontinua from highly nonlinear fibers by three-wave mixing
}

\author{
Philipp Sulzer, ${ }^{1, *} \odot$ Cornelius Beckh, ${ }^{1}$ Andreas Liehl, ${ }^{1}$ Jeldrik Huster, ${ }^{1}$ \\ Kilian R. Keller, ${ }^{1}$ Moritz Cimander, ${ }^{1}$ Philipp Henzler, ${ }^{1}$ ○ Christian Traum, ${ }^{1}$ \\ Claudius Riek, ${ }^{1}$ Denis V. Seletskiy, ${ }^{1,2}$ and Alfred Leitenstorfer ${ }^{1}$ \\ 'Department of Physics and Center for Applied Photonics, University of Konstanz, D-78457 Konstanz, Germany \\ ${ }^{2}$ Department of Engineering Physics, Polytechnique Montréal, Montréal, Quebec H3T 1J4, Canada \\ *Corresponding author: philipp.sulzer@uni-konstanz.de
}

\begin{abstract}
The nonlinear transformation of fluctuations by frequency broadening is found to produce strong anti-correlations in the spectral output. This effect is investigated by dispersive Fourier transform measurements. We exploit the anti-correlations in order to cancel the intensity noise in a subsequent sum-frequency mixing step. This principle allows for the generation of tunable visible pulses by cascaded nonlinear mixing whilst maintaining the same intensity noise performance as the input pulses. In addition, we demonstrate that the power fluctuations occurring in the process of passive stabilization of the carrier-envelope phase locking via difference frequency generation may be cancelled by an analogous strategy.
\end{abstract}

Experiments with ultrafast time resolution typically rely on generation of stable pulse trains by mode-locked lasers and subsequent nonlinear frequency conversion. In this context, high repetition rates in the megahertz range provide excellent statistics but come with relatively low pulse energies. Under those conditions, broadband and flexible frequency shifting is implemented by dispersion-managed nonlinear fibers [1]. Up till now, most experiments in ultrafast optics have been analyzed in terms of average values measured for a specific physical quantity. Recently, however, an increasing number of time-domain studies highlights higher statistical moments of time-domain observables, thus illuminating nontrivial fluctuations of classi$\mathrm{cal}$ and quantum degrees of freedom [2-7]. Facilitating further progress on this new frontier requires precise characterization of the noise properties of nonlinearly transformed pulse trains on a pulse-by-pulse basis. In particular, the coherence properties of supercontinua generated by nonlinear fibers have been studied extensively in a manifold of different regimes $[8,9]$. However, efficient strategies for minimizing and even exploiting any spectral correlations of noise in the emission from such elements is lacking so far.
In this Letter, we identify amplified spectral intensity fluctuations in the supercontinuum generated by highly nonlinear fibers (HNFs). These deviations are shown to be strongly anti-correlated and cause the output to slightly differ from a coherent state. The correlations are studied numerically resulting in quantitative agreement with the experiments. Based on this comprehensive understanding, we demonstrate how the increase of relative intensity noise (RIN) may be passively eliminated by an additional second-order nonlinear mixing step. This goal is achieved by judicious choice of phase-matching conditions and spectral shape of the HNF output. In this way, we implement generation of low-noise pulses tunable in the visible range. Finally, we present establishment of a passively phasestabilized frequency comb by difference frequency mixing [10], which may even surpass the intensity noise performance of the fundamental laser source.

The Er:fiber laser systems used in the following are comprised of a mode-locked oscillator followed by single-pass amplifiers, see Fig. 1(a). The pulse repetition rate was $100 \mathrm{MHz}$ for the noise cancellation in the difference frequency generation (DFG) and $40 \mathrm{MHz}$ in all other measurements. The resulting output pulses at a center wavelength of $1.55 \mu \mathrm{m}$ have a duration of $100 \mathrm{fs}$ and a pulse energy of more than $4 \mathrm{~nJ}$. They are launched into an $\mathrm{HNF}$ assembly generating an ultrabroadband spectrum [11]. These devices consist of a standard single-mode fiber with a core diameter of $8 \mu \mathrm{m}$ spliced to a few-millimeter-long germanosilicate fiber with a core diameter of $4 \mu \mathrm{m}[12,13]$. The nonlinear dynamics in these fibers may be quantitatively modeled, solving the generalized nonlinear Schrödinger equation. Resulting spectra are shown in Fig. 1(b) versus input pulse energy. Soliton fission and the emission of multiple dispersive waves limit the broadening. The process is dominated by the instantaneous Kerr nonlinearity, providing a maximally deterministic response to input fluctuations. A high degree of coherence is obtained when working with nonlinear components as short as half a period of the higher-order soliton [14]. Intrapulse Raman scattering and uncontrolled cascaded fission cause excess noise in longer specimens [1]. 

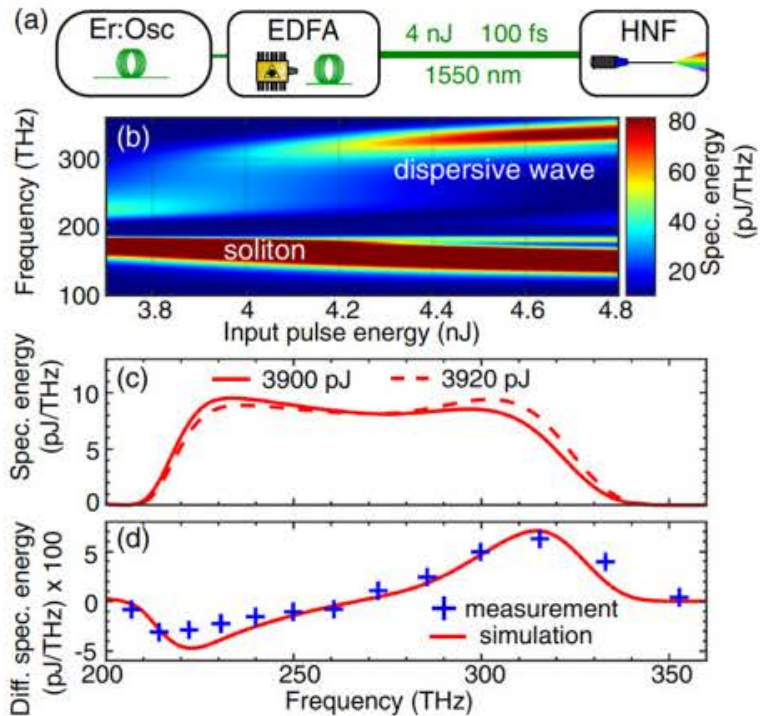

Fig. 1. (a) Sketch of the Er:fiber front-end with a subsequent highly nonlinear fiber (HNF). Er:Osc, mode-locked Er:fiber oscillator; EDFA, femtosecond Er:fiber amplifier. (b) Color-coded spectral energy density of supercontinua generated in an HNF for varying input pulse energy. (c) Simulated spectral energy density of a dispersive wave for two input pulse energies (solid and dashed lines). (d) Change in spectral energy density (red line) due to energy fluctuations of the input pulses by $0.05 \%$, corresponding to an energy difference of 2 pJ. Blue crosses indicate data obtained by spectrally resolved measurements.

Owing to the nonlinear propagation in the HNF, a continuous blue and red shift of dispersive-wave $(>200 \mathrm{THz})$ and solitonic $(<190 \mathrm{THz})$ components follows from increasing the pulse energy, respectively. Consequently, small amplitude fluctuations of the input are expected to cause characteristic spectral shifts of the HNF output, even if they originate from quantum effects. To investigate these phenomena, we first characterize the stability of the pulse energy emitted by the Er:oscillator-amplifier system. Integration of its RIN power spectral density from $100 \mathrm{~Hz}$ to the Nyquist frequency results in root-mean-square (RMS) shot-to-shot fluctuations as low as $5 \times 10^{-4}$. Nevertheless, such tiny input variations induce a minute shift in the peak positions of soliton and dispersive waves. To quantify these effects, we compare the spectra calculated for slightly different pulse energies. The solid and dashed lines in Fig. 1(c) correspond to dispersive waves generated with $3.9 \mathrm{~nJ}$ and $3.92 \mathrm{~nJ}$ pulses, respectively. Note that for illustrative purposes the level of intensity deviations has been set one order of magnitude higher as compared to the experiment where the energy fluctuations amount to $2 \mathrm{pJ}$ only. In Fig. 1(d), the differential spectral changes expected for a level of input RMS amplitude fluctuations of $5 \times 10^{-4}$ are depicted as a red graph. The measured level of amplitude noise at each spectral position, denoted by blue crosses, underlines the quantitative agreement. Note that the negative sign of the experimental data at frequencies below $270 \mathrm{THz}$ has been inferred at this point. Interestingly, a spectrally integrated characterization of the amplitude noise of the dispersive wave yields close to shot-noise-limited performance, indicating a fully coherent character of the emission.
When spectrally resolving the noise distributions, however, significant deviations from the standard quantum limit are found. This seeming contradiction is reconciled, when the anti-correlated character of the spectral noise demonstrated in Figs. 1 (c) and $1(\mathrm{~d})$ is taken into account.

We now study these noise correlations by implementing a real-time spectral readout of the supercontinua via dispersive Fourier transform [15], as depicted in Fig. 2(a). After the HNF, the dispersive wave [similar to the ones shown in Fig. 1(b)] is compressed to a duration of $5.8 \mathrm{fs}$ by a sequence of three SF 10 Brewster prisms [2]. A razor blade (RB) inserted in the Fourier plane removes the fundamental and solitonic parts of the full supercontinuum below $200 \mathrm{THz}$. We then couple the output into $79 \mathrm{~m}$ of a single-mode dispersion-compensating fiber (DCF, Nufern Inc., FUD-3034) with a second-order dispersion of $\beta_{2}=50 \mathrm{ps}^{2} / \mathrm{km}$. Propagation through the DCF stretches the pulses to a duration of 4 ns. A neutral density filter (ND) set in the path of the low-frequency components through the prism compressor attenuates their spectral weight. This step compensates for the strong losses of high-frequency light in the fiber. We record the pulses in the time domain using a fast photodetector (Newport Inc., New Focus 1577A, $12 \mathrm{GHz}$ ) and real-time oscilloscope (Rhode \& Schwarz GmbH, RTO1044, $4 \mathrm{GHz}$ and $20 \mathrm{GSa} / \mathrm{s}$ ). This configuration provides a spectral resolution of $10 \mathrm{THz}$. Measurement of the pulse spectrum with an optical spectrum analyzer (OSA) allows for a calibrated time-to-frequency mapping with a linear transfer function, consistent with dominant second-order dispersion in the DCF. Subsequently, we detect consecutive spectra emitted from the $\mathrm{HNF}$ and compute an intensity correlation map [16,17]. It is made up of Pearsons correlation coefficients calculated between pairs of spectral intensities ranging between -1 and 1 . The degree of correlation between the photocurrents measured for any combination of frequencies is color coded in Fig. 2(b). Note that the correlation coefficients were corrected by disattenuation [18] for the influence of uncorrelated contributions stemming from photodetector dark noise and digitization artifacts in the real-time oscilloscope. This procedure allows us to estimate the actual strength of correlation. It also increases the error margins, thus leading to values exceeding one close to the diagonal. The blue-colored rectangular regions on this map depict anti-correlations between high- and low-frequency components of the dispersive wave, consistent with the findings in Fig. 1. Red colors indicate positive correlation between the spectral regions. The white line overlaid onto the correlation map displays the average spectral energy density on a linear scale. Figure 2(c) shows horizontal cuts through the map at $220 \mathrm{THz}$ and $320 \mathrm{THz}$, respectively. We find coefficients as strong as -0.8 , consistent with strong anti-correlations. This fact underlines the deterministic character of the spectral fluctuations in the supercontinuum.

Can the precise understanding of spectrally correlated intensity fluctuations be exploited to passively suppress excess noise in subsequent frequency-mixing processes? To investigate this intriguing question, we now consider the sum-frequency generation (SFG) process excited by a compressed dispersive wave in a 4-mm-thick periodically poled lithium niobate (PPLN) crystal [19]. Figures 3(a) and 3(b) show the spectral energy density and its change (red lines) expected for a typical shot-to-shot fluctuation of the pump energy in close analogy to Figs. 1(b) and $1(\mathrm{c})$. The phase-matching condition is only fulfilled for a 


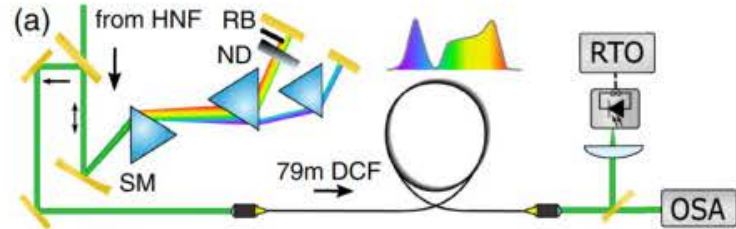

(b) SED $\quad-1$ Correlation 1 (c)

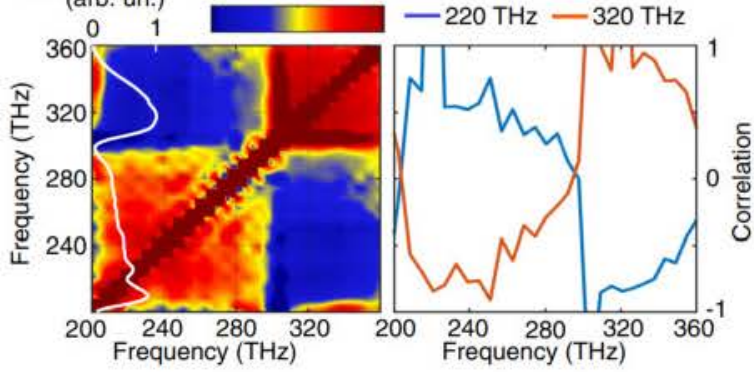

Fig. 2. (a) Experimental setup for dispersive Fourier transform measurements consisting of a three-prism compressor and dispersioncompensating fiber (DCF). HNF, highly nonlinear fiber; ND, neutral density filter; RB, razor blade; SM, spherical mirror; OSA, optical spectrum analyzer; RTO, real-time oscilloscope. (b) Colorcoded frequency-frequency correlation map. Correlations and anti-correlations are indicated by red and blue colors, respectively. The periodic structures visible around the positive diagonal originate from discretization of the data at the spectral resolution of $10 \mathrm{THz}$. White curve (left): spectral energy density (SED) of dispersive wave versus frequency. (c) Spectral noise correlations at $220 \mathrm{THz}$ (blue) and $320 \mathrm{THz}$ (red) as a funcrion of frequency.

small range of output frequencies around a center frequency $v_{\mathrm{SHG}}$. In this regime, SFG leads to narrowband spectral output in the visible range, as depicted in Fig. 3(c). Note that not only fundamental components at half the output frequency $v_{\mathrm{SHG}} / 2$ [dashed vertical line in Figs. 3(a) and 3(b)] contribute to the nonlinear mixing. Instead, SFG between components symmetrically detuned from $v_{\mathrm{SHG}} / 2$ contributes efficiently and over a large bandwidth $[20,21]$. The gray- and red-shaded areas in Figs. 3(a) and 3(b) correspond to the contributing spectral components, respectively. The relative position of $v_{\mathrm{SHG}} / 2$ inside the broadband dispersive wave is determined by the poling period of the PPLN, which is easily tunable due to its fan-out geometry [see inset in Fig. 3(c)]. We now analyze the RIN spectra of several upconverted pulses using a radio-frequency spectrum analyzer, see Fig. 3(d). Pulses at $v_{\text {SHG }}$ of $470 \mathrm{THz}$ and $545 \mathrm{THz}$ (orange and blue, respectively) exhibit a significantly increased noise level, as compared to the RIN of the input fluctuations (black). In both cases, the corresponding values of $v_{\mathrm{SHG}} / 2$ are well above or below the spectral center of the dispersive wave. Therefore, components with mostly correlated noise contribute to the SFG and result in a drastic increase of fluctuations. In contrast, at $\nu_{\mathrm{SHG}} / 2=260 \mathrm{THz}$ (green graph), the contributions from anti-correlated spectral segments are similar in magnitude and lead to a remarkable all-passive suppression of noise of the visible output pulses. This case is close to the situation sketched in Fig. 3(b). The balance of anti-correlated input fluctuations leads to a noise performance comparable to the one of the Er:fiber amplifier output. This fact proves a virtual absence of degradation in the RIN of the upconverted pulses after two nonlinear
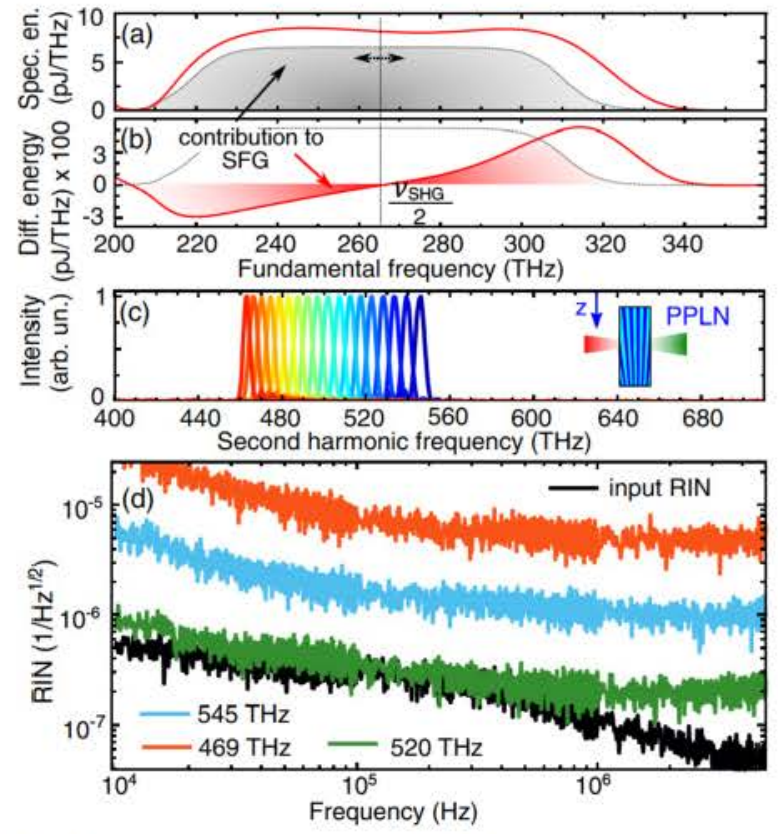

Fig. 3. (a) Simulation of the spectral energy density (red lines) of a dispersive wave generated in an HNF. The black line and grayshaded area indicate components contributing to SFG for a specific PPLN poling period. (b) Difference in spectral energy density due to typical fluctuations of HNF input power. The red-shaded area denotes contributions to the nonlinear mixing process. (c) Spectra of the narrow-band visible pulses generated via SFG. The inset sketches a PPLN with a fan-out design, where translation enables continuous adjustment of the quasi-phase-matched frequencies. (d) RIN spectra for different target frequencies $v_{\text {SHG }}$. The RIN of the HNF input pulses is depicted in black.

conversion stages. The supercontinuum generation process itself may be tuned by changing the chirp or the average pulse energy of the input pulses [12]. Therefore, it is straightforward to use these degrees of freedom in combination with the tunable quasi-phase matching to generate low-noise emission of second harmonic across the full available bandwidth.

We also implement a similar scheme based on DFG, allowing for generation of longer wavelengths, e.g., in the mid infrared. Moreover, this step passively eliminates the carrier-envelope offset frequency and provides an inherently phase-stable frequency comb $[10,22,23]$. Here, a slightly different design of the HNF assembly based on a fiber with a smaller zero-dispersion wavelength maximizes the bandwidth of the supercontinuum [12]. This adjustment results in a soliton and a dispersive wave centered around $160 \mathrm{THz}$ and $350 \mathrm{THz}$, respectively. As before, the intensity noise is first simulated numerically, as depicted in Figs. 4(a) and 4(b), in close analogy to Figs. 1(c) and 1(d). We use a PPLN crystal for DFG between parts of the ultrabroad spectrum and select specific components to be involved in this process via the poling period. The dotted black lines in Figs. 4(a) and $4(\mathrm{~b})$ indicate the selected frequencies and $v_{\text {disp }}$ denotes the center frequency of the dispersive-wave contribution. The redshaded areas in Fig. 4(b) show the noise features that lead to RIN reduction in the DFG process because of integration over anticorrelated spectral regions. We now vary the contribution from 
the dispersive wave by setting its center frequency $v_{\text {disp }}$ through changes in the poling period of the nonlinear crystal. Owing to the flat dispersion of lithium niobate around $150 \mathrm{THz}$, the contribution from the soliton to the DFG process is independent of the poling period. The resulting evolution of the RIN is plotted as a function of $v_{\text {disp }}$ in Fig. 4(c). Filled circles denote the RIN measured at frequencies of $6 \mathrm{kHz}$ (blue), $70 \mathrm{kHz}$ (magenta), and $1.5 \mathrm{MHz}$ (red) as a function of $v_{\text {disp. }}$. A pronounced minimum occurs at an optical input frequency of $340 \mathrm{THz}$, where the noise performance even surpasses the output levels of the Er:fiber front end, which are indicated by color-coded horizontal lines. At this frequency position, the effects of anti-correlated input fluctuations balance out optimally, and our measurement becomes limited by electronic detection noise. The results are in excellent agreement with our numerical simulations, which are depicted as dashed colored lines in Fig. 4(c).

In summary, we have studied the spectral noise properties of the output of versatile HNF assemblies in both experiment and simulation, showing pronounced (anti-)correlations over the entire output bandwidth. The insights are exploited to suppress the intensity fluctuations by nonlinear mixing of strongly anti-correlated spectral regions. This capability is crucial in the context of passively phase-stable frequency combs, where DFG in a carefully tailored supercontinuum provides elimination of both the carrier-envelope phase and intensity excess noise in one elegant step. Also, the noise properties of widely tunable spectra

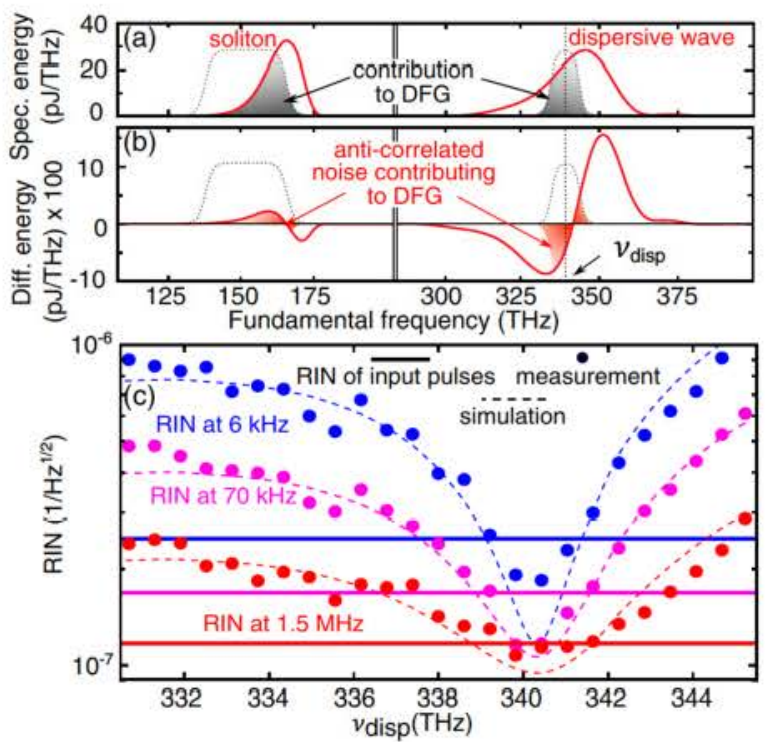

Fig. 4. (a) Simulation of the spectral energy density (red) of soliton (left) and dispersive waves (right). Black dotted lines indicate spectral regions with phase matching for DFG, producing the gray-shaded areas contributing to the process. The center frequency of the contribution from the dispersive wave is denoted as $v_{\text {disp }}$ (vertical dotted line). (b) Difference in spectral energy density (red lines) due to intensity fluctuations of the input for soliton and dispersive waves, respectively. Red-shaded segments indicate spectral weight and sign of correlated noise contributions to the DFG process for a configuration close to exact cancelation. (c) RIN levels of radiation generated via DFG versus $v_{\text {disp. }}$. Filled circles show measurements in various frequency bands. Results of a numerical simulation and RIN levels of the seed are depicted as dashed and solid lines with analogous color coding, respectively. in the visible, which are generated by SFG from a broadband input in the near infrared, may be optimized by this strategy. Furthermore, our results are the key to advancing a new class of ultrafast measurements where the noise has become the signal.

Funding. European Research Council (290876).

Acknowledgment. This work was supported by TOPTICA Photonics AG. We thank Alexander Rieber for helpful discussions on statistical analysis.

Disclosures. The authors declare no conflicts of interest.

\section{REFERENCES}

1. J. M. Dudley, G. Genty, and S. Coen, Rev. Mod. Phys. 78, 1135 (2006).

2. C. Riek, D. V. Seletskiy, A. S. Moskalenko, J. F. Schmidt, P. Krauspe, S. Eckart, S. Eggert, G. Burkard, and A. Leitenstorfer, Science $\mathbf{3 5 0}$, 420 (2015).

3. I.-C. Benea-Chelmus, C. Bonzon, C. Maissen, G. Scalari, M. Beck, and J. Faist, Phys. Rev. A 93, 043812 (2016).

4. C. Riek, P. Sulzer, M. Seeger, A. S. Moskalenko, G. Burkard, D. V. Seletskiy, and A. Leitenstorfer, Nature 541, 376 (2017).

5. F. Randi, M. Esposito, F. Giusti, O. Misochko, F. Parmigiani, D. Fausti, and M. Eckstein, Phys. Rev. Lett. 119, 187403 (2017).

6. I.-C. Benea-Chelmus, F. F. Settembrini, G. Scalari, and J. Faist, Nature 568, 202 (2019).

7. J. O. Tollerud, G. Sparapassi, A. Montanaro, S. Asban, F Glerean, F Giusti, A. Marciniak, G. Kourousias, F. Billè, F. Cilento, S. Mukamel, and D. Fausti, Proc. Natl. Acad. Sci. USA 116, 5383 (2019).

8. N. R. Newbury, B. R. Washburn, K. L. Corwin, and R. S. Windeler, Opt. Lett. 28, 944 (2003).

9. M. Klimczak, G. Soboń, K. Abramski, and R. Buczyński, Opt. Express 22, 31635 (2014).

10. D. Fehrenbacher, P. Sulzer, A. Liehl, T. Kälberer, C. Riek, D. V. Seletskiy, and A. Leitenstorfer, Optica 2, 917 (2015).

11. A. Sell, G. Krauss, R. Scheu, R. Huber, and A. Leitenstorfer, Opt. Express 17, 1070 (2009).

12. D. Brida, G. Krauss, A. Sell, and A. Leitenstorfer, Laser Photon. Rev. 8, 409 (2014).

13. A. Liehl, P. Sulzer, D. Fehrenbacher, S. Eggert, M. Ludwig, F, Ritzkowsky, D. V. Seletskiy, and A. Leitenstorfer, Phys. Rev. A 101, $023801(2020)$.

14. S. Kumkar, G. Krauss, M. Wunram, D. Fehrenbacher, U. Demirbas, D. Brida, and A. Leitenstorfer, Opt. Lett. 37, 554 (2012).

15. P. V. Kelkar, F. Coppinger, A. S. Bhushan, and B. Jalali, Electron. Lett. 35, 1661 (1999).

16. B. Wetzel, A. Stefani, L. Larger, P. A. Lacourt, J. M. Merolla, T. Sylvestre, A. Kudlinski, A. Mussot, G. Genty, F. Dias, and J. M. Dudley, Sci. Rep. 2, 882 (2012).

17. T. Godin, B. Wetzel, T. Sylvestre, L. Larger, A. Kudlinski, A. Mussot, A. B. Salem, M. Zghal, G. Genty, F. Dias, and J. M. Dudley, Opt. Express 21, $18452(2013)$

18. C. Spearman, Am. J. Psychol. 15, 72 (1904)

19. C. Traum, P. Henzler, S. Lohner, H. Becker, D. Nabben, P. Gumbsheimer, C. Hinz, J. F. Lippmann, S. Mahapatra, K. Brunner, D. V. Seletskiy, and A. Leitenstorfer, Rev. Sci. Instrum. 90, 123003 (2019).

20. K. Moutzouris, F. Adler, F. Sotier, D. Träutlein, and A. Leitenstorfer, Opt. Lett. 31, 1148 (2006).

21. M. Marangoni, D. Brida, M. Quintavalle, G. Cirmi, F. M. Pigozzo, C. Manzoni, F. Baronio, A. D. Capobianco, and G. Cerullo, Opt. Express 15, $8884(2007)$

22. A. Liehl, P. Sulzer, D. Fehrenbacher, T. Rybka, D. V. Seletskiy, and A. Leitenstorfer, Phys. Rev. Lett. 122, 203902 (2019).

23. E. Benkler, B. Lipphardt, T. Puppe, R. Wilk, F. Rohde, and U. Sterr, Opt. Express 27, 36886 (2019) 